\title{
Survey of Collaborative Data Mining
}

\author{
$1^{\text {st }}$ Carmen Ana Anton \\ Electric, Electronic and Computer \\ Engineering Department, \\ Technical University of Cluj-Napoca, \\ North University Center Baia Mare, \\ Baia Mare, Romania \\ carmen.anton@cunbm.utcluj.ro
}

\author{
$2^{\text {nd }}$ Oliviu Matei \\ Electric, Electronic and Computer \\ Engineering Department, \\ Technical University of Cluj-Napoca, \\ North University Center Baia Mare, \\ Baia Mare, Romania \\ oliviu.matei@cunbm.utcluj.ro
}

\author{
$3^{\text {rd }}$ Anca Diana Avram \\ Electric, Electronic and Computer \\ Engineering Department, \\ Technical University of Cluj-Napoca, \\ North University Center Baia Mare, \\ Baia Mare, Romania \\ anca.avram@ieee.org
}

\begin{abstract}
In the information industry there is a huge amount of data which can be used to obtain relevant information necessary for them to be processed, cleaned and analyzed. This survey paper presents different data extraction research that can be applied in various areas. The main point is to determine a new approach and to be the starting point for new experiments in agriculture predictions. The collaborative data mining approach assumes that correlated units or devices will have similar behavior that can be determined with an acceptable approximation.
\end{abstract}

Keywords—data mining, collaborative, correlated data, virtual machine learning

\section{INTRODUCTION}

Extracting information is a complex process involving a number of activities such as data cleansing, data integration, data transformation, data mining, evaluation and integration of data and presentation of information in context.

The process of extracting the information is interesting and challenging, but not without problems that may arise along the way (missing data, number and identification of relevant characteristics, few or too many data etc.). However, as new applications appear every challenge creates opportunities for new research and innovative solutions. Factors that can lead to new opportunities are considered by $\mathrm{O}$. Matei in [14] as:

- The size of scientific data sets.

- The emergence of new computer architectures.

- Analyzing new data types.

- The need to analyze real-time data.

- Analysis of geographically distributed data.

- The need to draw conclusions in the presence of uncertainty.

Extracting information is not an easy task, but the multitude of application fields make it attractive. The current trend of development in the context of Industry 4.0, is the automation of data and exchange technology determination. Modern technology, which uses algorithms to generate information, are increasingly better able to detect and address issues that can be resolved in a faster and more efficient way, for example, maintenance and repair, product degradation, wear components etc.

In this context, the basic characteristic intended, by big companies, are to opening new fields of research directions and customization and adaptation problems as quickly as possible.

\section{ETYMOLOGY AND BACKGROUND}

\section{A. Data Mining in General}

The concept of data mining is defined as an extraction of relevant knowledge from massive data or as virtual machine learning. A more precise definition may be "process of analyzing large volumes of data and extract useful and relevant information, using various statistical methods, mathematical and algorithmic" given by $\mathrm{B}$. Lika and $\mathrm{K}$. Kolomvatsos in [2].

A more appropriate definition is given by Grinstein et al. [6], data mining is a "mechanized process of identifying or discovering useful structure in data." By structure, the authors understand patterns, models or relations over the data.

Now, we are surrounded by data that invade our world and life. Information that we have, numbers, images, sounds, is stored and saved, and push us to postpone decisions. But, the Internet gives companies information about our preferences and influence decisions in industry, commerce, national security etc. This leads to a series of questions, such as: How to select optimal and relevant information, quickly? How do we discover behavior patterns in the data available? How do we protect personal information? The list could continue, but all lead to a new field of study, namely that of extracting information from massive data. To understand the full process, is necessary a brief overview of some areas addressed successfully to date.

Starting from examples such as weather forecasting, determining the type of contact lenses needed for a person, finding the identification rules for diagnosing diseases of soybean, Witten, Frank, Hall and Pal in [16] provides a ranking of applications to virtual machines learning:

- Web mining: search engines extract information from web content (publicity received). Books, movies, recommended products based on previous preferences are options encountered in digital space. 
- Decisions involving judgement: getting a loan based on a questionnaire applied is a practice of the industry. Interesting cases are those of the limit that can not be analyzed too easy. For them, applying a machine learning process can get classification rules with success rates attractive and profitable.

- Screening images: detecting oil slicks or environmental disasters using images provided by satellites.

- Load forecasting: forecasting demand for electricity in order to create reserves, maintenance scheduling, and fuel inventory management.

- Diagnosis: a field of expert systems.

- Marketing and sales: is the most active domain which uses data mining and for which the prediction is very useful. Banks, mobile phone companies, supermarkets use large-scale KDD (knowledge discover information) and applying pieces of informations discovered.

Until today, in many different fields have been approached techniques of machine learning. Results were obtained with applications in biology, biomedicine, chemistry, ecology, national security, etc., some even rivaling with those of human experts. In a world full of data, we are obliged to adapt and to learn new ways to detect useful information. "Machine learning is used to detect intrusion by recognizing unusual patterns of operation." as it was defined by Chakrabarti Soumen et al. in [4].

\section{B. Collaboration in Data Mining}

Collaboration as a general term comes from active participation through an effective contribution to a party, to work jointly to an end. In data mining, it is meet this concept in many forms: collaborative filtering, collaborative networks etc.

One technique widely used in e-commerce and the internet is the "Collaborative Filtering". It is a method that addresses the following issues: people often choose, based on recommendations from somebody with similar tastes to themselves. Thus, collaborative filtering techniques include matching people with similar interests making recommendations based on this premise.

In the next representation, Fig. 1 and Fig. 2, of collaborative filtering we highlight the similarities between users or products according to the case presenting.

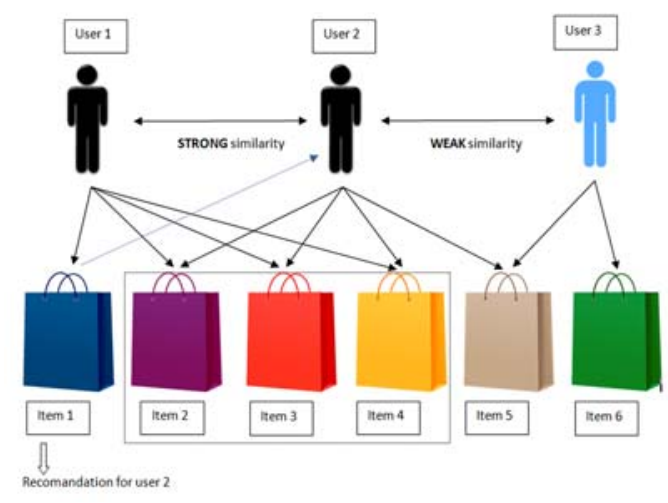

Fig. 1 Proposed user-based collaborative filtering

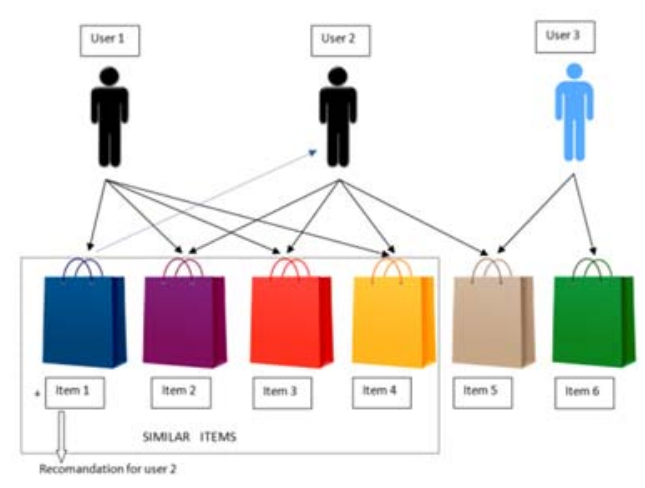

Fig. 2 Proposed item-based collaborative filtering

Another aspect of the term collaboration is meet at networking collaborative presented by Camarinha-Matos et al. in [9], which was defined as a "network of entities which are largely autonomous, but they collaborate for purposes compatible or better, and whose interactions are a supported network ". The idea that emerges is that the collaboration intentional give better results, less expensive and with forecasts able to be discovered.

Because data mining involves searching and finding relevant information from massive data, why do not we might think of "collaboration" of multiple entities that conduct us to next concept: two sources of data, correlated with each other, to be able to provide relevant information just by analyzing one of them, which would lead to a prediction of behavior for the second source. We might expand this concept to multiple interconnected sources, and we obtain a new notion of multiple collaborative data mining.

The concept of data mining collaborative, presented and demonstrated by $\mathrm{O}$. Matei et al. in [12], reveals that two sources which are correlated, will grow the accuracy of data that need to be extracted and for data that are not correlated will lower the accuracy of extracting information. Collaboration in the case of the two relevant sources allows and generated a domain of study and open the window to probe this concept in various areas of applicability.

\section{RESEARCH IN DOMAIN}

As is mentioned in [15] by S. Moyle, data mining effort for collaborative data mining, it is larger and divided between all participants in the process.

Addressing the notion of collaborative in the mining process would imply a correlation between certain limits for the participants in the process. A collaborative process could be studied from several points of view: use of 2 or 3 sources, followed by a generalization. To predict the behavior of one source we try to use the data from multiple sources in a virtual machine learning process.

Extracting information from data is a complex process that involves a phased approach. Collaboration could express that participating actively in an operation whereby each party brings its effective contribution. L.M. Camarinha-Matos and $\mathrm{H}$. Afsarmanesh in [8], define collaborative as an act whereby a variety of entities share information, resources and responsibilities to plan, implement and evaluate a joint program of activities, all targeting the same goals, and enumerating the requirements for its success: 
- the main purpose of collaboration;

- the initial baseline for collaboration;

- the collaborative process (a set of generic steps);

- the creation of a space for collaboration;

- and finally the definition of resources, rewards, commitment, and responsibilities.

With all these considered, the proposed research is based on the assumption that if there are similarities between devices/ agents studied, this could lead to sustainable use models, said by B. Sanou in [3]. In such a way, we propose a data mining model that takes into account the conduct of other devices/ agents when conducting similar patterns recognition.

Data mining research is needed to address more collaborative than two devices/ agents correlations as different or distinct intervals. Furthermore, a comparison between a non-collaborative approach and one based collaboration could highlight how behaviors are affected and which ones have a higher success rate.

Finally, considering the growing number of data with multiple similarities study is required to allow a generalized representation of them and to come quickly and accurately processes of extracting information with methodological same track.

Addressing the context of each application should be considered and may generate a new perspective on the problem and working context.

For a collaborative data mining process, it would be useful to study two, three or more sources and find a relationship between the extracted information and the correlation of the source data.

Data mining of collaboration can occur in any area of research, but of course, each case must be analyzed according to its specificity. As a starting point, a common approach is needed to allow the extraction process data follow the same semantic and syntax given for each of the contexts in which they arise. Many factors that determine and characterize a procedural data mining it may create some problems.

One of the major challenges encountered in the domain of extracting information is the quality of the data and process that are being applied. The data can come from different sources can have different representations and be complete or not. In these circumstances, the challenges posed by data mining techniques are not only data and methods used to extract information, but also process the data available so as to provide as many accurate results.

Steps in data processing can be summarized in the following list given by F. Guillet and J. Hamilton in the [5]:

- data selection or data sets or objects;

- selection for variable weights and characteristics;

- data encoding or recoding;

- missing data analysis and sources;

Measuring the success of a process of data mining can be achieved using multiple sizes or factors involved, namely: accuracy, precision, robustness, scalability, and interpretability of the model's applicability in various cases.
The accuracy may be defined in several ways and from many perspectives.

1. Accuracy can be how exact that is done an action or an object.

\section{Accuracy is opposite of the notion of error.}

3. Accuracy can be interpreted as the probability that the result obtained is correct.

4. Accuracy is the ratio between the numbers of positive examples correctly classified and the total number of examples, given by I. H. Witten et al. in [16].

Matei et al. in [13] have developed an extensible data extraction model which, through their detailed analysis and application of various algorithms, can bring useful knowledge about the use of machinery in the shoe production line industry and the level of accuracy of predictions according to input/ output data.

The robustness of a process is given by the effective ways of treating those missing or incomplete data or incorrect. Applicability to process large data sets its scalability and give a general interpretation and an applicable model.

A data mining process that is based on accurate and complete data-quality information will provide reliable and well-documented information.

\section{COLlaborative DATA Mining}

The term of collaborative data mining has been used several times, by Appleman in [1], Maimon in [10] and Moyle in [15]. But the term is not defined in a comprehensive manner that allows one to have a view of what are the advantages of such an approach compared to classical data mining, what are the benefits of such an approach, what are the underlying principles of such an approach and how this approach may be different compared to other approaches. Later on, in 2008, Zhan et al. [17] used the term collaborative as referring to using more data sources for mining.

When we want to obtain a data mining process as accurately as possible, we need, in addition to classical data, to adapt and understand the context of their use and add the data that can positively influence the process and increase the accuracy of the results.

A very significative step in the data mining process is to establish connections between data, relationships that could lead to a more accurate prediction. A possibility to establish these relationships is to measure the correlations between the variables involved in the process.

In English Oxford Living Dictionaries the term correlation means "a mutual relationship or connection between two or more things or the process of establishing a relationship or connection between two or more things."1 A more appropriate definition for this paper is ,the tendency for two values or variables to change together, in either the same or opposite way." 2 Han et al. [7] use the Pearson's correlation coefficient to establish the correlation between two variables $\mathrm{X}$ and $\mathrm{Y}$. If we have two data sets for $\mathrm{X}$ and $\mathrm{Y}$, respectivily $\left\{\mathrm{x}_{1}, \ldots, \mathrm{x}_{\mathrm{n}}\right\}$ for $\mathrm{X}$ and $\left\{\mathrm{y}_{1}, \ldots, \mathrm{y}_{\mathrm{n}}\right\}$ for $\mathrm{Y}$, the formula for $\mathrm{r}$ (sample correlation coefficient) is:

\footnotetext{
${ }^{1} \mathrm{https}$ ://en.oxforddictionaries.com/definition/correlation
}

${ }^{2}$ https://www.thefreedictionary.com/correlation 


$$
r_{x y}=\frac{\sum_{i=1}^{n}\left(x_{i}-\bar{x}\right)\left(y_{i}-\bar{y}\right)}{(n-1)\left(s_{x} s_{y}\right)}
$$

where: $\mathrm{n}$ is the number of samples, $\bar{x}$ and $\bar{y}$ are the samples means of $\mathrm{X}$ and $\mathrm{Y}$, and $s_{x}$ and $s_{y}$ are the corrected sample standard deviations of $\mathrm{X}$ and $\mathrm{Y}$.

Correlation is a statistical technique that can show whether and how strongly pairs of attributes are related.

A correlation is a number between -1 and +1 that measures the degree of association between two attributes ( $\mathrm{X}$ and $\mathrm{Y})$. The numbers in the interval $[-1,1]$ can be explained like this:

Case 1: If the number is higher than 0 , closer to +1 , the semnification is that the correlation between two attributes is strong, and the correlation is positive.

Case 2: If the result is 0 it means that two attributes are independent and there is no correlation.

Case 3: If the number is less than 0 , closer to -1 , semnification is that the correlation between two attributes is also strong, and the correlation is negative.

Data mining correlations can be viewed as an additional analysis to find interesting links or dependencies between sets of elements to determine whether they have a (positive, negative, or none) effect on each other. Analyzing data sets can lead to their classification and predictive modeling to set predictable behaviors with a small margin of error.

The application of this processing phase can be used in concrete experiments and can be the starting point for determining better and more accurate results if the correlations are positive or negative.

O. Matei et al. in [11] present a data mining system capable of gathering weather data from several weather stations and predicting the moisture of the soil for the next day, all in real time. The system has been tested in real-life conditions, in Transylvanian Plain, with ten weather stations. They have proven that the prediction accuracy is very high and the system can be used in agriculture as a mature platform in any geo-climate conditions.

A prior knowledge of the characteristics of the soil, temperature, precipitation levels, moisture content, soil type, etc. may decisively influence the success or failure of one culture and can make the difference in a society which have increasing need of food.

\section{CONCLUSION AND FUTURE WORK}

One of the actual challenges of the world is global warming. Modifications of the climate are felt by society and have a great importance in many areas. Agriculture felt hard this change and the research in the field try to adapt the process and actions to support this industry.

Predicting temperature and humidity values could be an asset for agriculture. A different approach to data mining in this area could be successful, so experiments on a collaborative approach to data from multiple sources are in progress, and future work will present the results.
There are several tools on the market or in the research phase for predicting weather at various levels of accuracy and detail. Most of the algorithms use stand-alone data sources. However, combining, fusing or making use of third party data sources have proven a powerful approach in increase the accuracy of the forecasts, especially when they have specific scope, such as agriculture or aviation.

\section{REFERENCES}

[1] Appleman, Kenneth H., et al. "Collaborative internet data mining systems." U.S. Patent No. 5,918,010. 9 Jun. 1999.

[2] Blerina Lika, Kostas Kolomvatsos, Stathes Hadjiefthymiades, "Facing the cold start problem in recommender systems", Expert Systems with Applications, volume 41, march 2014.

[3] Brahima Sanou, "ITC Facts and Figures 2013", Telecommunication Development Bureau, International Telecommunications Union (ITU), Geneva, February 2013. Retrieved 23 May 2015.

[4] Chakrabarti, Soumen, et al. Data mining: know it all. Morgan Kaufmann, 2008.

[5] Fabrice Guillet, Howard J. Hamilton (Eds.), "Quality Measures in Data Mining”, Springer, 2007.

[6] Grinstein, Georges G., Andreas Wierse, and Usama Fayyard. "Information visualization in data mining and knowledge." (2002).

[7] Han, Jiawei, Jian Pei, and Micheline Kamber. Data mining: concepts and techniques. Elsevier, 2011.

[8] L. M. Camarinha-Matos and H. Afsarmanesh, Eds., "Collaboration forms," in Collaborative Networks: Reference Modeling, Springer US, 2008, pp. 51-66.

[9] L. M. Camarinha-Matos, M. Luis, and Hamideh Afsarmanesh. "Collaborative networks: a new scientific discipline." Journal of intelligent manufacturing 16.4-5 (2005): 439-452.

[10] Maimon, Oded, and Lior Rokach, eds. Data mining and knowledge discovery handbook. Vol. 2. New York: Springer, 2005.

[11] Matei, Oliviu, et al. "A data mining system for real time soil moisture prediction." Procedia Engineering 181 (2017): 837-844.

[12] Oliviu Matei, Giovanni Di Orio, Javad Jassbi, José Barata, Claudio Cenedese, "Collaborative Data Mining for Intelligent Home Appliances", Proc. Of PRO-VE, Springer, 2016.

[13] Oliviu Matei, Kevin Nagorny and Karsten Stoebener, "Applying data mining in the context of Industrial Internet" International Journal of Advanced Computer Science and Applications(IJACSA), 7(1), 2016. http://dx.doi.org/10.14569/IJACSA.2016.070184.

[14] Oliviu Matei, Preliminary Results Of The Analysis Of Field Data From Ovens, Carpathian Journal of Electrical Engineering, vol. 8, no. 1, 2014.

[15] S. Moyle, "Collaborative Data Mining," in Data Mining and Knowledge Discovery Handbook, O. Maimon and L. Rokach, Eds. Springer US, 2009, pp. 1029-1039.

[16] Witten, Ian H., et al. Data Mining: Practical machine learning tools and techniques. Morgan Kaufmann, 2016.

[17] Zhan, Justin. "Privacy-preserving collaborative data mining." Computational Intelligence Magazine, IEEE 3.2 (2008): 31-41. 